\title{
Glutathione-Related Systems and Modulation of Extracellular Signal-Regulated Kinases Are Involved in the Resistance of AGS Adenocarcinoma Gastric Cells to Diallyl Disulfide-Induced Apoptosis
}

\author{
Giuseppe Filomeni, Katia Aquilano, Giuseppe Rotilio, and Maria R. Ciriolo \\ Department of Biology, University of Rome "Tor Vergata," via della Ricerca Scientifica, Rome, Italy
}

\begin{abstract}
We have previously characterized the cytotoxic action of diallyl disulfide (DADS) on neuroblastoma cells, and we have shown the crucial role of an early and massive reactive oxygen species production in the induction of c-Jun $\mathrm{NH}_{2}$-terminal kinase-mediated apoptotic pathway. In the present work, we report that DADS is ineffective in inducing apoptosis in a human adenocarcinoma gastric cell line (AGS). In particular, we show that AGS cells are able to recover from the p53/p21mediated cell cycle arrest in the $G_{2}$-M phase upon DADS treatment without committing cells to death. This event is most likely due to a peculiar surviving pathway of these cells involving: (a) the formation of mixed disulfides between reduced glutathione (GSH) and protein thiols, (b) a higher and inducible glutathione peroxidase activity, and/or (c) an efficient modulation of the phospho-active levels of the extracellular signal-regulated kinases 1 and 2 (ERK 1/2). Moreover, by increasing glutathione peroxidase expression or GSH concentrations, cell cycle arrest is fully abolished; the apoptotic death is induced by either decreasing the availability of intracellular GSH or inhibiting the reactivation of ERK 1/2. Altogether, our data show that ERK $1 / 2$ participates in the active proliferation of AGS cells and that an efficient reactive oxygen species buffering system makes these cells resistant to DADS-mediated detrimental effects. (Cancer Res 2005; 65(24): 11735-42)
\end{abstract}

\section{Introduction}

Several cellular functions are the results of oxidation-reduction (redox) activities, among which mitochondrial respiration (1), cytosolic oxidase, and oxygenase activities $(2,3)$ are responsible for the reduction of oxygen leading to reactive oxygen species (ROS). Redox unbalance in favor of oxidation may result in a condition of oxidative stress that induces specific cellular responses. To minimize adverse effects resulting from excessive production of ROS, cells have elaborated defense systems that include enzymes and low molecular weight antioxidants (4). Particularly, the reduced glutathione (GSH)/oxidized glutathione (GSSG) redox couple provides a very efficient system in maintaining the intracellular redox environment (5). The majority of GSH is found in the cytosolic compartment, where it is synthesized and present

Requests for reprints: Maria R. Ciriolo, Department of Biology, University of Rome "Tor Vergata" via della Ricerca Scientifica, 00133 Rome, Italy. Phone: 39-06-7259-4374; Fax: 39-06-7259-4311; E-mail: ciriolo@bio.uniroma2.it.

(C)2005 American Association for Cancer Research.

doi:10.1158/0008-5472.CAN-05-3067 at a concentration ranging from 1 to $11 \mathrm{mmol} / \mathrm{L}$. The oxidized isoforms of this tripeptide are mainly represented by the intramolecular disulfide GSSG, the levels of which are 100- to 1,000-fold lower than those of GSH, and by the less abundant mixed disulfides between GSH and protein thiols (GS-R; refs. 5, 6).

Besides the induction of oxidative damage on proteins (7), phospholipids (8), and DNA (9), ROS are also able to function as second messengers by activating specific signal transduction terminating as a cell response leading to death in a gene-programmed manner (apoptosis; refs. 4, 10).

A chemotherapeutic and/or chemopreventive use of phytochemicals could open new avenues in searching for strategies against the proliferation of cancer cells. Among these compounds, redox-active molecules, such as polyphenols from grapes, berry fruits, and sulfurcontaining compounds from garlic seem to be good candidates for a direct or a combined approach in cancer treatment. On one side, the low concentrations of these molecules seem to be able to counteract the deleterious effects of environmental stresses associated with oxidative injury (such as UV radiation, smoke, diet, etc.; refs. 11-14). On the other hand, a therapeutic use for high concentrations of these molecules could help in selectively killing tumor cells through sustained and site-directed bursts of ROS $(15,16)$. In fact, the chemotherapeutic action of several anticancer drugs commonly used (e.g., Adriamycin) is partly based on their capability to catalyze one-electron redox cycles with oxygen, thus producing high concentration of ROS, capable of selectively killing tumor cells (17).

Several biological regulatory systems are redox-sensitive; among them, signal transduction pathways mediated by the activation of mitogen-activated protein kinases (MAPK) are well-established examples (18-20). We have previously observed that diallyl disulfide (DADS) increases intracellular levels of ROS (21). The increased steady-state concentration of ROS is sensed by glutathione transferase that, through its oxidatively induced detachment from c-Jun $\mathrm{NH}_{2}$-terminal kinase (JNK), leaves the JNK/c-Jun signal transduction pathway free to autoactivate and initiate apoptosis via the mitochondrial route (21).

DADS is one of the most abundant oil-soluble garlic derivatives, with several beneficial properties. It has been reported that DADS can decrease the risk of hypertension and coronary diseases owing to its inhibitory action on plaque formation (22) and on cholesterol synthesis (23). Moreover, it has been shown that DADS has both antioxidant and anticancer properties and that both functions are dependent on its concentration. A low concentration of DADS could be a potential therapeutic or modulating agent for neurodegenerative and other diseases associated with oxidative damage, whereas high concentrations could be a powerful tool against proliferation, through the induction of apoptosis in cancer cells. 
However, cancer cells are differently equipped against ROS production. In fact, it has been suggested that increased proliferation could be a consequence of an increased ROS flux, which may result from impairment of the antioxidant cell system (24-26). Thus, it is reasonable that cancer cells could be differently susceptible to ROS-mediated detrimental damage.

In this study, we showed that a human adenocarcinoma gastric cell line (AGS) is characterized by an unusually high level of GS-R and glutathione peroxidase (GPx). Concomitantly, these cells undergo a transient growth arrest but are resistant to DADSinduced apoptosis even at very high DADS concentrations. Moreover, we found a specific modulation of extracellular signalregulated kinases 1 and 2 (ERK 1/2) pattern that could explain the transitory and protective arrest of cell cycle without commitment to apoptosis.

\section{Materials and Methods}

Cell culture. Human adenocarcinoma cells (AGS) were purchased from the American Type Cell Culture Collection (Manassas, VA) and were grown in F12 medium supplemented with $10 \%$ FCS, at $37^{\circ} \mathrm{C}$ in an atmosphere of $5 \% \mathrm{CO}_{2}$ in air. Cells were routinely trypsinized, plated at $4 \times 10^{4} / \mathrm{cm}^{2}$ flasks. Cell viability was assessed by trypan blue exclusion.

Treatments. A solution of $50 \mathrm{mmol} / \mathrm{L}$ DADS (Sigma, Co., St. Louis, MO) was prepared just before the experiments dissolving $5.5 \mathrm{~mol} / \mathrm{L}$ DADS in DMSO. Treatments were done with different amounts of DADS ranging from 10 to $200 \mu \mathrm{mol} / \mathrm{L}$ at $37^{\circ} \mathrm{C}$ in medium supplemented with serum. Unless specified, the concentration of DADS selected for all the experiments was $50 \mu \mathrm{mol} / \mathrm{L}$ because it gives a valuable degree of apoptosis on SH-SY5Y neuroblastoma cells and because it is in the range used for in vivo study (21). As control, an equal amount of DMSO (0.1\%) was added to untreated cells.

D,L-Buthionine $S, R$-sulfoximine (BSO) was used at the final concentration of $1 \mathrm{mmol} / \mathrm{L}$ to reduce GSH neo-synthesis by the irreversible inhibition of $\gamma$-glutamate-cysteine ligase activity and was maintained in culture medium for 12 hours. Then AGS cells were washed with fresh medium and treatments with DADS were done.

GSH ethyl ester (GSHest) was supplemented to the culture medium at a concentration of $2 \mathrm{mmol} / \mathrm{L}$ for 6 hours; then the medium was removed to avoid nonspecific reaction between GSHest and DADS, just before treatments with DADS.

Selenomethionine (Sigma) was used at a final concentration of $0.5 \mu \mathrm{mol} / \mathrm{L}$ to enhance the intracellular concentration of selenium that is normally associated with an increase in the expression levels of the seleniumcontaining family of enzyme Gpxs (27). This concentration was selected on the basis of efficacy in the induction of the enzymes and inefficiency in being cytotoxic.

Treatment with the cell-permeable MEK $1 / 2$ inhibitor, U0126 (Calbiochem-Novabiochem, La Jolla, CA) was done at a concentration of $0.26 \mu \mathrm{mol} / \mathrm{L}$ because lower concentrations did not show significant inhibition of ERK 1/2 phosphorylation, and higher concentrations were toxic. U0126 was added 1 hour before the addition of DADS and was maintained throughout the experiment. Detection of intracellular ROS was done as previously described (21).

Analysis of cell viability and apoptosis. Adherent (after trypsinization) and detached cells were combined, washed in PBS, and counted after trypan blue staining, directly by optical microscope on hemocytometer. Alternatively, cells were stained with $50 \mu \mathrm{g} / \mathrm{mL}$ propidium iodide (dissolved in $0.1 \%$ Triton X-100) prior to analysis by a FACScalibur instrument (Becton Dickinson, San Jose, CA). The percentages of cells in each phase of the cell cycle were evaluated according to Nicoletti et al. (28) by calculating peak areas of nuclei with different amounts of DNA.

Preparation of cell lysates and Western blot analyses. Cell pellet was resuspended in lysis buffer containing $10 \mathrm{mmol} / \mathrm{L}$ Tris- $\mathrm{HCl}$ (pH 7.4), $5 \mathrm{mmol} / \mathrm{L}$ EDTA, $150 \mathrm{mmol} / \mathrm{L} \mathrm{NaCl}, 0.5 \%$ IGEPAL CA-630, and protease inhibitors (Sigma). Proteins $(20 \mu \mathrm{g})$ were transferred onto a nitrocellulose membrane after SDS-PAGE. Polyclonal anti-GPx $(1: 1,000)$, a gift from Prof. Marmocchi, University of Camerino, Italy, anti-phospho-p $38^{\mathrm{MAPK}}$, phospho-ERK 1/2, p42/44 (1:1,000; Cell Signaling, Beverly, MA), anti-p21, anti-p38 ${ }^{\mathrm{MAPK}}$, anti-ERK and anti-JNK $(1: 1,000)$, and monoclonal antiphospho-activated JNK (1:1,000; Santa Cruz Biotechnology, Santa Cruz, CA), anti-p53 and $\beta$-actin (1:5,000; Sigma) were used as primary antibodies. The specific protein complex, formed upon specific secondary antibody treatment (1:10,000; Bio-Rad, Hercules, CA), was identified using a Fluorchem Imaging system (Alpha Innotech, Analitica De Mori, Italy) after incubation with ChemiGlow chemiluminescence substrate (Alpha Innotech).

Glutathione determination and other assays. Intracellular glutathione was assayed upon formation of $S$-carboxymethyl derivatives of free thiols with iodoacetic acid, followed by the conversion of free amino groups to 2,4-dinitrophenyl derivatives by the reaction with 1-fluoro-2,4-dinitrobenzene as previously described (20). Cell lysates were divided into two aliquots: one used for GSH/GSSG assay, whereas the other was used to determine mixed disulfides by treating with $300 \mathrm{mmol} / \mathrm{L} \mathrm{NaBH}_{4}$ at $40^{\circ} \mathrm{C}$ for 30 minutes prior to derivatization, as previously described (29). Data are expressed as nanomoles of GSH equivalents per milligram of protein.

Glutathione peroxidase activity. Total GPx activity was measured spectrophotometrically as previously described (30), following the decrease of absorbance at $340 \mathrm{~nm}$, using $\mathrm{H}_{2} \mathrm{O}_{2}$ as substrate. Data are expressed as nanomoles of NADPH consumed per minute per milligram of protein.

Measurement of oxidative damage. Carbonylated proteins were detected using the Oxyblot Kit (Intergen, Purchase, NY) after reaction with 2,4-dinitrophenylhydrazine, as previously described (21). Levels of malondialdehyde and 4-hydroxynonenal were measured as previously described (21) by a colorimetric method using the Lipid Peroxidation Assay Kit (Calbiochem-Novabiochem). Proteins were determined by the method of Lowry et al. (31).

Data presentation. All experiments were done at least five different times $(n=5)$ unless otherwise indicated. The results are presented as means \pm SD. Statistical evaluation was conducted by ANOVA, followed by correction with Bonferroni's test. Comparisons were considered to be significant at $P<0.05$.

\section{Results}

Diallyl disulfide treatment induces a transient cell cycle arrest in AGS cells. The effects of DADS on AGS cells proliferation were analyzed by direct counts upon trypan blue exclusion. Treatment was done with $50 \mu \mathrm{mol} / \mathrm{L}$ DADS on cells plated at a density of $4 \times 10^{4} / \mathrm{cm}^{2}$, as previous experiments carried out on neuroblastoma cells, SH-SY5Y, showed that these conditions were successful in inducing apoptosis (21). Cell growth, monitored for up to 60 hours of treatment, shows that DADS induced an arrest of proliferation, particularly evident between 12 and 24 hours of treatment (Fig. 1A), without a concomitant increase of trypan blue-positive cells (data not shown). This is indicative of a lack of cytotoxic effects under the experimental conditions employed and was confirmed by the efficient recovery of cell growth evidenced at longer time intervals, when the number of viable cells treated with DADS almost matched the control values (Fig. $1 A$ ). To further evaluate the effects of DADS on growth arrest, we treated AGS cells plated at a lower density $\left(2 \times 10^{4} / \mathrm{cm}^{2}\right)$ with $50 \mu \mathrm{mol} / \mathrm{L}$ of DADS. As depicted in the inset of Fig. $1 A$, this condition amplified the cytostatic effect of DADS, but cells, although at later time points ( $>96$ hours), recovered the capability to proliferate (data not shown). We next did a cytofluorimetric analyses of the cell cycle by staining AGS cells with propidium iodide. Histograms depicted in Fig. $1 B$ (and values in the table reported below), show that DADS treatment was able to induce a time-dependent increase of the percentage of cells in $\mathrm{G}_{2}-\mathrm{M}$ phase with a maximum effect at 
Figure 1. DADS induces p53/p21-dependent cell cycle arrest but not apoptosis in AGS cells. A, AGS cells were plated at a density of $4 \times 10^{4} / \mathrm{cm}^{2}$ and treated with $50 \mu \mathrm{mol} / \mathrm{L}$ DADS. At indicated time points, cells were harvested and counted upon trypan blue staining. Alternatively, the experiment was done at a density of $2 \times 10^{4}$ cells $/ \mathrm{cm}^{2}$ (inset). Points, mean of viable cells only (trypan blue-negative); bars, $\pm \mathrm{SD} ;{ }^{* *}, P<0.01(n=6)$. $B$, AGS cells were treated with $50 \mu \mathrm{mol} / \mathrm{L}$ DADS up to 48 hours, washed and stained with propidium iodide. Analysis of cell cycle and apoptosis was done by a FACScalibur instrument and the percentages of cells in cell cycle phases were calculated using WinMDI version 2.8 software. The cell cycle histograms reported are from one of five typical experiments done in triplicate which gave similar results. A table indicating the percentages of nuclei in the sub- $G_{1}$ and $G_{2}-M$ phases of the cell cycle is shown at the bottom (sub- $\mathrm{G}_{1}$, apoptotic cells). C, AGS cells were treated with $50 \mu \mathrm{mol} / \mathrm{L}$ of DADS up to 24 hours. Total cell extracts $(20 \mu \mathrm{g})$ were loaded for detection of p53 and p21 expression levels. Immunoblots are from one of three representative experiments that gave similar results. $D$, AGS cells were treated with $50 \mu \mathrm{mol} / \mathrm{L}$ DADS for 24 hours. After treatment, cells were stained with propidium iodide for cell cycle and apoptosis cytofluorimetric analyses. Percentages of sub- $\mathrm{G}_{1}$ and $\mathrm{G}_{2}-\mathrm{M}$-arrested cells were calculated using WinMDI version 2.8 software. Columns, means; bars, $\pm \mathrm{SD}(n=5)$; *, $P<0.01 ;{ }^{*}, P<0.001$

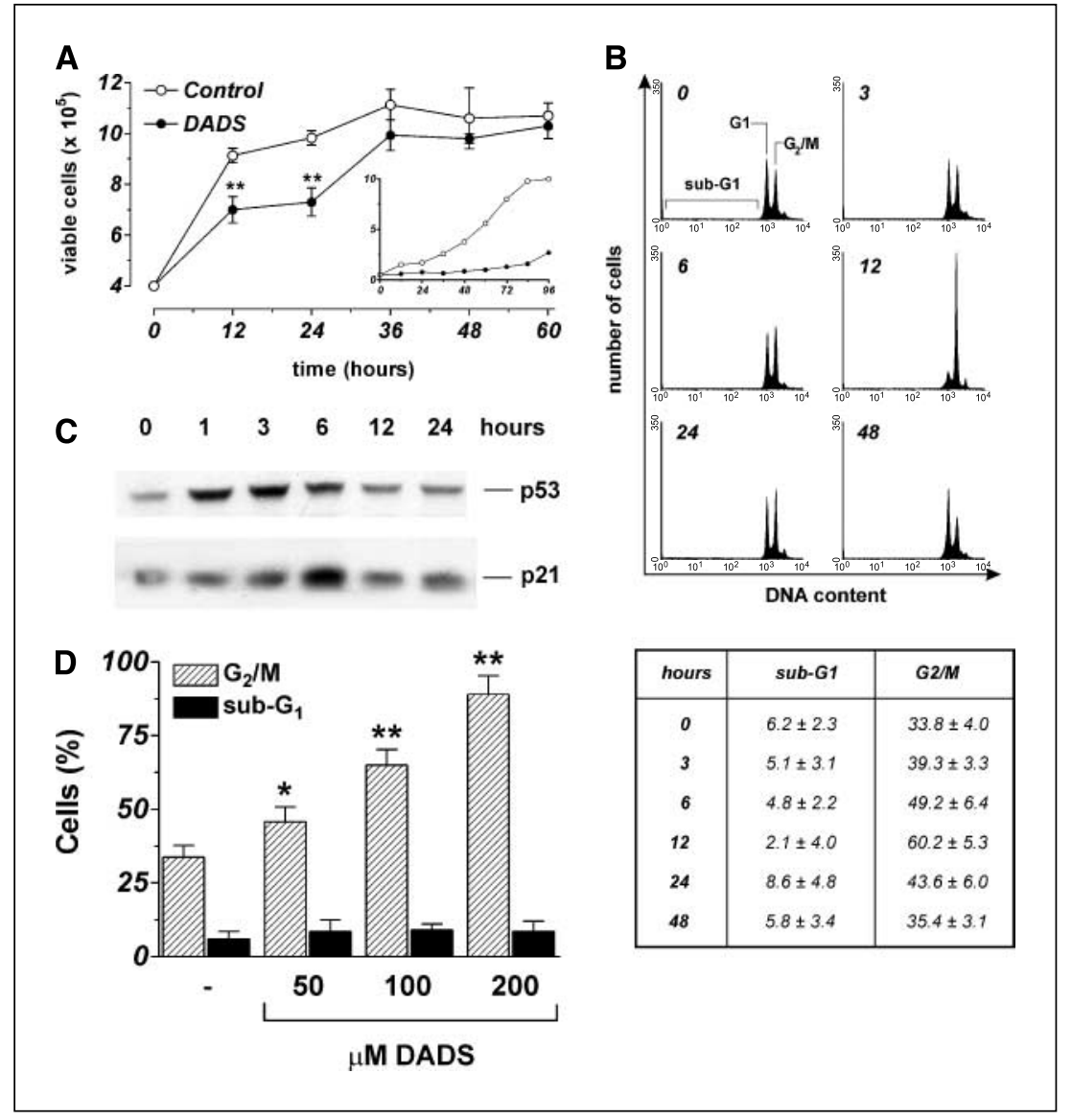

12 hours $(60.2 \pm 5.8 \%$ versus $33.8 \pm 4.0 \%$ calculated in untreated cells). Moreover, we found a slight increase of the percentage of sub- $G_{1}$ (apoptotic) cell population after 24 hours of treatment. However, this phenomenon was followed by a recovery of the cell cycle progression at 48 hours of treatment, demonstrating that AGS cells were able to counteract DADS-mediated toxicity. Thus, we did Western blot analyses of the main molecular factors involved in the inhibition of cell cycle progression, p53 and p21. Figure $1 C$ shows that the expression levels of p53 rapidly increased between 1 and 3 hours of treatment with DADS, followed by a slow decrease up to 6 hours, to finally reach the control values at 12 and 24 hours. As a downstream response, p21 protein levels were raised in a time-dependent manner up to 6 hours, returning to the basal concentration at 12 and 24 hours (Fig. $1 C$ ). In order to show that the observed effects were not a mere consequence of a low dose of DADS employed, we did cytofluorimetric analyses of AGS cells treated for 24 hours with 100 and $200 \mu \mathrm{mol} / \mathrm{L}$ of DADS. Figure $1 D$ shows that higher doses of DADS did not result in a further increase of the percentage of sub- $G_{1}$ cell population but only strengthened the cytostatic effect, as the time of cell cycle recovery shifted to 96 hours (data not shown).

Reduced glutathione-related systems are implicated in the resistance of AGS to diallyl disulfide treatment. We previously showed that, in neuroblastoma cells, ROS production represented the earliest causative event of the apoptosis induction triggered by DADS (21). In order to evaluate whether the different responses of AGS cells was due to a lower induction of oxidative stress, we first examined the intracellular concentration of ROS by cytofluorimet- ric analyses, using the fluorochrome DCF-DA. As evidenced in Fig. $2 A$, only a slight increase in the percentage of DCF-positive cells was observed upon treatment with DADS $(\sim 3.5 \%$ between 10 and 30 minutes), which rapidly declined after 1 hour.

To confirm the capacity of AGS cells in buffering DADSmediated intracellular oxidative stress, we determined the content of protein oxidation in terms of carbonyl group formation through Western blot analyses, and by measuring the content of malondialdehyde and 4-hydroxynonenal, the extent of lipid peroxidation. Under our experimental conditions, we were not able to detect any significant changes either in protein carbonyls or in lipid peroxidation at different time points, ranging from 1 to 24 hours (data not shown).

The absence of a massive flux of ROS, as well as of a ROSdependent damage, let us hypothesize that AGS cells could be equipped with an efficient antioxidant defense system. In this context, it is worthwhile to mention that gastric-derived cell lines, besides the well-known GPx, possess an additional isoform of the enzyme, referred to as the gastrointestinal one (GI-GPx; ref. 32). On the basis of these findings, we determined the GPx activity spectrophotometrically by using $\mathrm{H}_{2} \mathrm{O}_{2}$ as a substrate. Figure $2 B$ shows that these cells are equipped with a very high physiologic level of the enzyme, especially if compared with the almost undetectable levels of GPx measured in other cell types (e.g., neuroblastoma SH-SY5Y cells; data from our laboratory). Moreover, the enzyme activity was significantly increased $(\sim 75 \%)$ after 6 hours of treatment with DADS, and it was maintained at high levels up to 24 hours of treatment. 


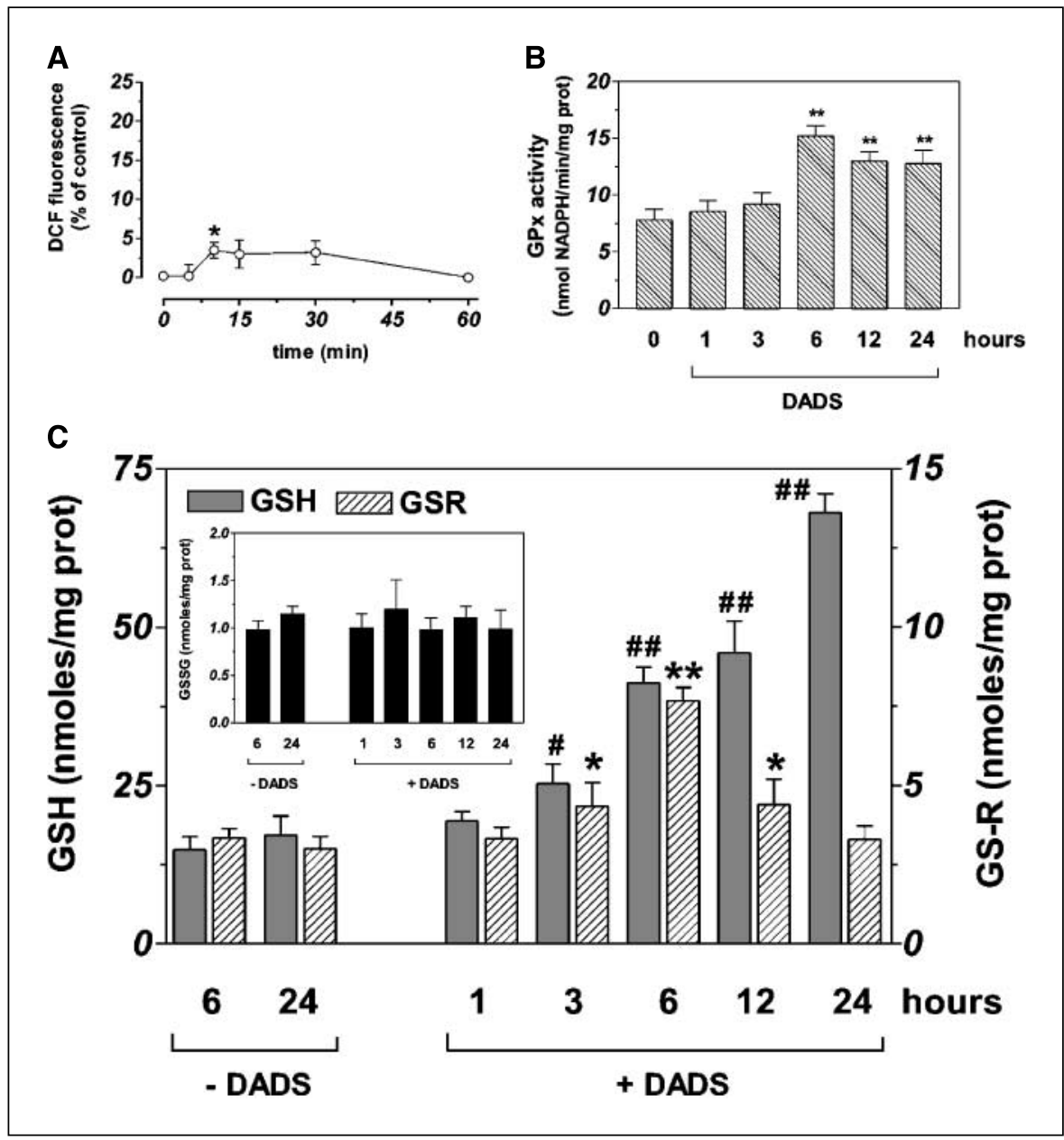

Figure 2. DADS-mediated oxidative insults are counteracted by GPx and GS-R formation. A, AGS cells were incubated with $50 \mu \mathrm{mol} / \mathrm{L}$ DCF-DA at $37^{\circ} \mathrm{C}$ for 30 minutes and then treated with $50 \mu \mathrm{mol} / \mathrm{L}$ DADS for up to 60 minutes. At the indicated time points, cells were washed with PBS and ROS production was analyzed by FACScalibur instrument. Points, means; bars, $+\mathrm{SD}(n=3) ;{ }^{*}, P<0.01$. B, AGS cells were treated with $50 \mu \mathrm{mol} / \mathrm{L}$ DADS for 24 hours. At the indicated time points, cells were harvested and lysed by sonication. GPx activity was detected by spectrophotometrically following the decrease of absorbance of NADPH at $340 \mathrm{~nm}$, as described in Materials and Methods. Data are shown as nmol of NADPH consumed $/ \mathrm{min} / \mathrm{mg}$ total protein; columns, means; bars, $\pm \mathrm{SD}(n=5)$; ${ }_{\star \star}, P<0.001$. C, AGS cells were treated with $50 \mu \mathrm{mol} / \mathrm{L}$ DADS up to 24 hours. At the indicated time points, cells were collected, exhaustively washed with PBS, and used for GSH, GSSG, and GS-R assay by HPLC as described in Materials and Methods. GSH, GSSG and GS-R data are expressed as nmol of GSH or GSSG/mg total protein; columns, means; bars, $\pm \mathrm{SD}(n=5)$. Significance was calculated with respect to each control: \#, $P<0.01$; \#\#, $P<0.001$ for GSH and ${ }^{*}, P<0.01 ;{ }^{*}, P<0.001$ for GS-R.

GSH represents the most important physiologic antioxidant either by directly reacting with ROS, or indirectly preserving cysteine residues of proteins from irreversible oxidations, giving rise to GS-R. Furthermore, it is the GPx cofactor for peroxidereducing reactions. We measured the content of intracellular GSH and its related redox species, in order to show a possible involvement of this redox system in the resistance of AGS cells to DADS treatment. Figure $2 C$ shows the GSH and GS-R levels analyzed by high-pressure liquid chromatography (HPLC) technique. Untreated cells showed an average intracellular GSH concentration of $\sim 12 \mathrm{nmol} / \mathrm{mg}$ protein, and, unusually, a well determinable concentration of GS-R $(4.02 \pm 0.65 \mathrm{nmol} / \mathrm{mg}$ protein). Cells treated with DADS showed a rapid and sustained increase of GSH levels $(68.04 \pm 4.12 \mathrm{nmol} / \mathrm{mg}$ protein $)$ up to 24 hours, most probably due to the detoxification of DADS (33). This phenomenon was associated with an increase of GS-R concentration $(7.67 \pm 0.82 \mathrm{nmol} / \mathrm{mg}$ protein) up to 12 hours, followed by a decline close to control values at 24 hours. Figure $2 C$ also shows the intracellular GSSG content that was not affected by treatment with DADS, remaining at very low levels, without significant changes with respect to control values ( $1 \mathrm{nmol} / \mathrm{mg}$ protein).

Modulation of the level of glutathione-related systems influences the effects of diallyl disulfide. In order to establish the role played by GSH and its redox-related species in the cellular response to DADS, we treated AGS cells with BSO, a specific inhibitor of $\gamma$-glutamate-cysteine ligase, to decrease the intracel- lular GSH levels. Figure $3 A$ shows that after 12 hours of incubation with $1 \mathrm{mmol} / \mathrm{L}$ BSO, AGS cells still presented detectable levels of GSH ( $-63 \%$ ) with values close to $4 \mathrm{nmol} / \mathrm{mg}$ of protein. The level of intracellular GS-R also declined with respect to untreated cells $(-49 \%)$, although to a lower extent. After 12 hours of incubation with BSO, cells were treated with $50 \mu \mathrm{mol} / \mathrm{L}$ of DADS for different times and then stained with propidium iodide for cytofluorimetric analyses. Figure $3 B$ shows that the GSH decrement made the cells prone to undergo apoptosis; in particular, the death process was time-dependent and reached values of $32.8 \pm 3.2 \%$ after 24 hours of treatment with DADS.

In order to further show the pivotal role of GSH and the related enzyme GPx in the resistance of AGS cells to DADS-mediated apoptosis, two different sets of experiments were done. First, we loaded the cells with the ethyl-ester form of GSH, which is able to freely cross the cell membrane enriching the intracellular GSH pool. Second, we induced the expression of GPx, by growing AGS cells for seven passages, in culture medium supplemented with 0.5 $\mu \mathrm{mol} / \mathrm{L}$ selenomethionine. Cells were treated with $50 \mu \mathrm{mol} / \mathrm{L}$ DADS and stained with propidium iodide for cytofluorimetric analyses. Figure $4 A$ shows that pretreatment with $2 \mathrm{mmol} / \mathrm{L}$ GSH-ethyl ester for 6 hours significantly decreased the percentages of the cell population arrested in $\mathrm{G}_{2}-\mathrm{M}$ phase upon treatment with $50 \mu \mathrm{mol} / \mathrm{L}$ DADS at 12 and 24 hours. This phenomenon was accompanied by an increase of the cells in $G_{1}$ phase, suggesting that, by raising intracellular GSH levels, AGS cells were more protected against 
DADS-mediated cell cycle arrest. Supplementation of $0.5 \mu \mathrm{mol} / \mathrm{L}$ selenomethionine induced an increase of GPx in terms of both activity and protein content (Fig. $4 B$ ). The enzyme activity was 3 -fold higher then untreated cells, and a comparable increment in the protein content was also detected. As shown in Fig. $4 C$, this condition allowed the cells to completely overcome the arrest in $\mathrm{G}_{2}-\mathrm{M}$ phase, giving rise to a superimposable trend of the GPX-overexpressing and untreated cells.

Diallyl disulfide treatment modulates expression of mitogenactivated protein kinases in AGS cells. DADS-induced apoptosis in neuroblastoma cells was mediated by the induction of the JNK-governed phosphorylative cascades. This event represented a downstream effect of ROS burst, because by preventing their production, the apoptotic response was inhibited (21). In order to characterize whether resistance of AGS cells to DADS could be associated with a different pattern of MAPK modulation, Western blot analyses of basal and phospho-activated isoforms of the proapoptotic members of the MAPK family, JNK, and $\mathrm{p} 38^{\mathrm{MAPK}}$ were done. Figure $5 A$ shows that no significant changes in the expression and phospho-activation levels of both JNK and p $38^{\mathrm{MAPK}}$ could be

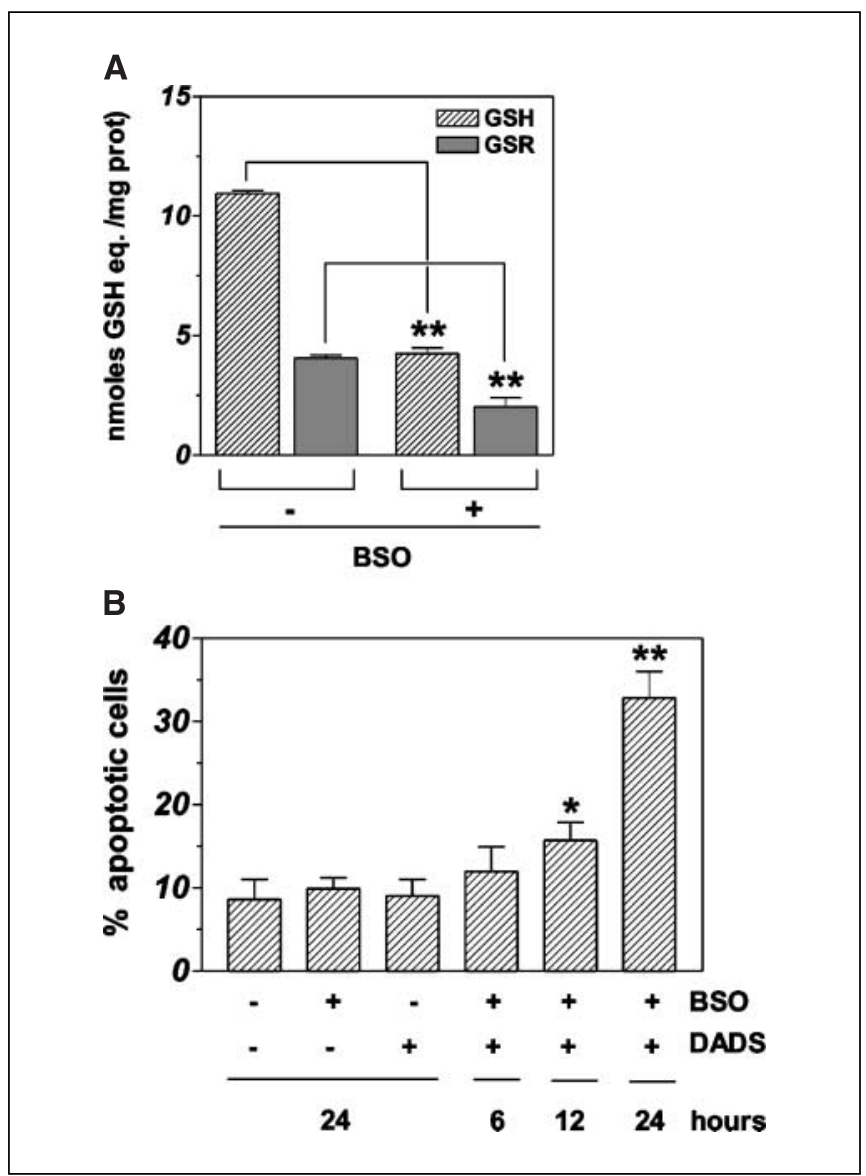

Figure 3. GSH depletion sensitizes AGS cells towards DADS-induced apoptosis. A, AGS cells were incubated with $1 \mathrm{mmol} / \mathrm{L}$ BSO for 12 hours and used for GSH and GS-R assay by HPLC as described in Materials and Methods. Data are expressed as nmol of GSH equivalents/mg total protein. Columns, means; bars, $\pm \mathrm{SD}(n=3) ;{ }^{\star *}, P<0.001$. $B$, AGS cells were incubated with $1 \mathrm{mmol} / \mathrm{L}$ BSO for 12 hours and then treated with $50 \mu \mathrm{mol} / \mathrm{L}$ of DADS for up to 24 hours. At each time point, cells were stained with propidium iodide for cytofluorimetric analyses. Percentages of sub-G ${ }_{1}$ (apoptotic) cells were calculated using WinMDI version 2.8 software. Columns, means; bars, $\pm \mathrm{SD}$ $(n=3) ;{ }^{*}, P<0.01 ;{ }^{* *}, P<0.001$.
A

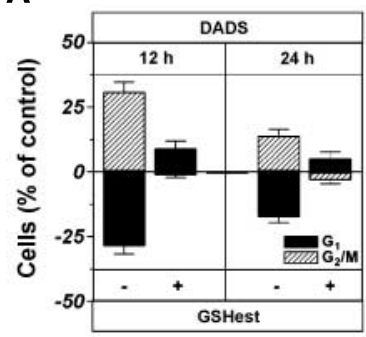

B

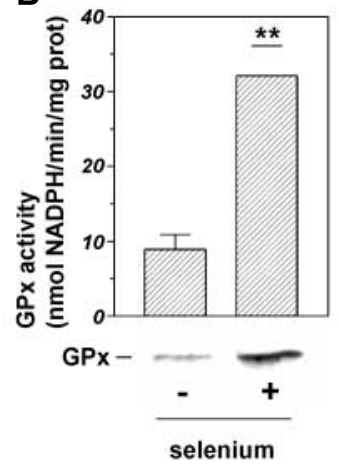

DADS

C

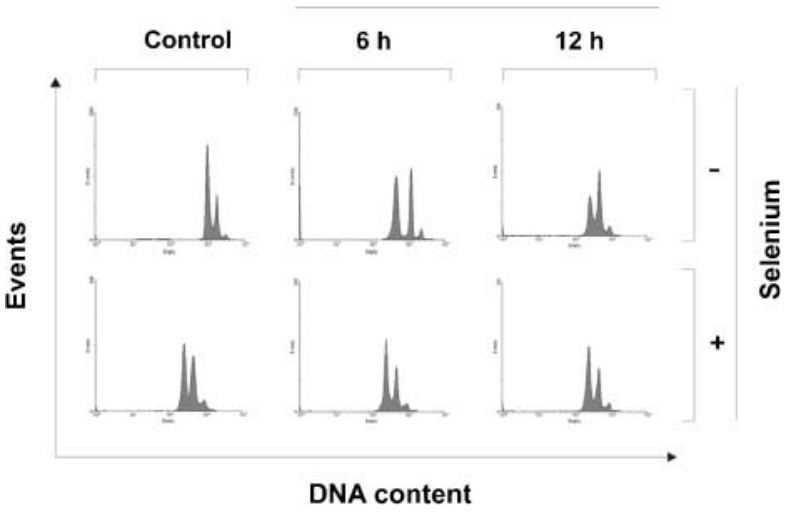

Figure 4. Increase of GSH levels or GPx activity rescues AGS cells from DADS-induced cell cycle arrest. $A$, AGS cells were incubated with $2 \mathrm{mmol} / \mathrm{L}$ GSHest for 6 hours, then treated with $50 \mu \mathrm{mol} / \mathrm{L}$ of DADS for up to 12 and 24 hours, and finally stained with propidium iodide for cytofluorimetric analyses. Percentages of the cells in the $\mathrm{G}_{1}$ and $\mathrm{G}_{2}-\mathrm{M}$ phases of cell cycle were calculated using WinMDI version 2.8 software. Columns, means; bars, $\pm \mathrm{SD}(n=3)$. All treated cells were significantly different with respect to controls $(P<0.001)$. $B$, AGS cells were grown for at least seven passages in medium containing $0.5 \mu \mathrm{mol} / \mathrm{L}$ selenomethionine to induce GPx expression. Then, cells were assayed for GPx activity by spectrophotometric analyses as described in Materials and Methods. Data are shown as nmol of NADPH consumed/min/mg total protein; columns, means; bars, $\pm \mathrm{SD}(n=5)$; ${ }^{*}, P<0.001$. Alternatively, Western blot analyses were done for the detection of GPx expression levels by loading $75 \mu \mathrm{g}$ of total cell extracts on SDS-PAGE. Immunoblots are from one of three representative experiments that gave similar results. $C$, AGS cells were grown for at least seven passages in medium containing $0.5 \mu \mathrm{mol} / \mathrm{L}$ selenomethionine to induce GPx expression. Then, cells were treated with $50 \mu \mathrm{mol} / \mathrm{L}$ of DADS for the following 6 and 12 hours, and finally stained with propidium iodide for cytofluorimetric analyses. The percentages of sub-G1 (apoptotic) cells were calculated using WinMDI version 2.8 software. The cell cycle plots reported are from one of five typical experiments done in triplicate that gave similar results.

determined. In particular, although phospho-JNK was also detected in high amounts in untreated cells, the phospho-active band of $\mathrm{p} 38^{\mathrm{MAPK}}$ was slightly evident, and no change was observed during treatment (Fig. $5 A$ ). ERK $1 / 2$ is another member of the MAPK family, which is related to the survival response. ERK 1/2-dependent phosphorylative cascades seem to be activated by oxidative stimuli as well, suggesting that ROS may have a regulatory role in its mitogenic activity. To completely evaluate the MAPK network in AGS cells, we determined the expression and activation level of ERK $1 / 2$ by Western blot analyses. As shown in Fig. $5 A$, the phospho-levels of ERK $1 / 2$ were easily detectable under resting conditions; moreover, they had already decreased at 3 hours 


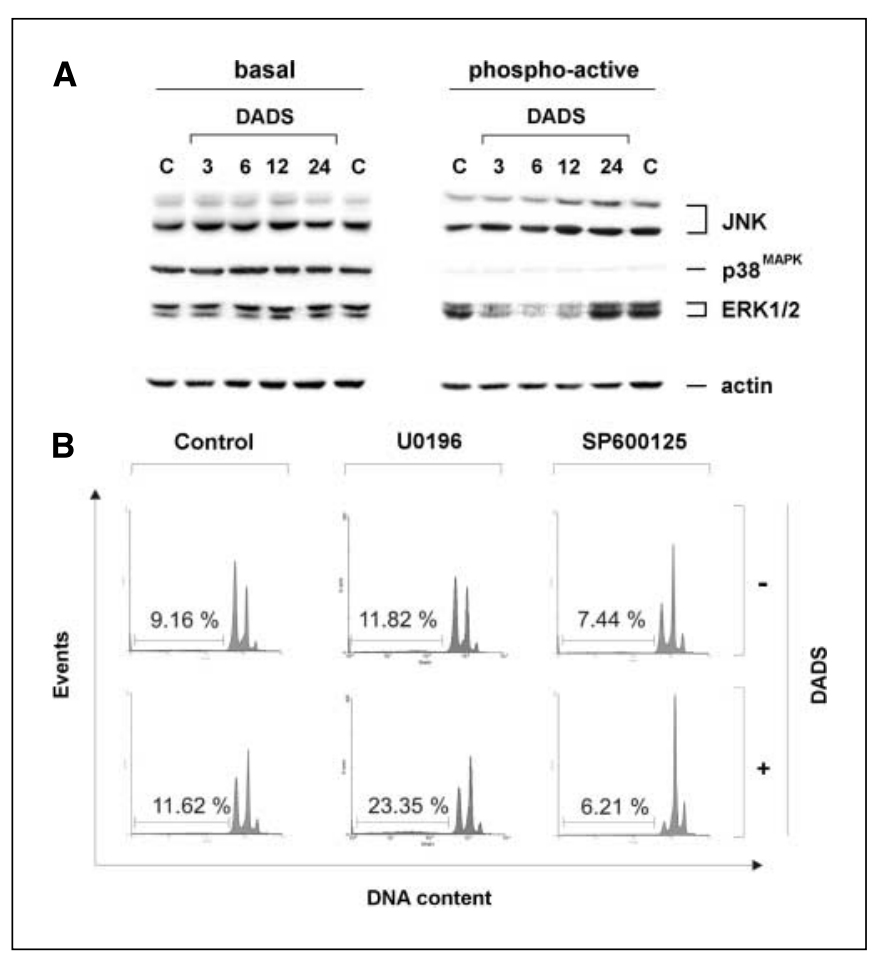

Figure 5. DADS treatment induces dephosphorylation of ERK 1/2. A, AGS cells were treated with $50 \mu \mathrm{mol} / \mathrm{L}$ DADS up to 24 hours. Total cell extracts $(20 \mu \mathrm{g})$ were loaded for detection of both the basal and phospho-active isoforms of JNK, p38 $8^{\mathrm{MAPK}}$, and ERK 1/2. Actin was used as a loading control. Immunoblots are from one of five representative experiments that gave similar results. $B$, AGS cells were incubated for 1 hour with U0126 or SP600125, the specific inhibitors of ERK $1 / 2$ and JNK, respectively. Then, cells were treated with $50 \mu \mathrm{mol} / \mathrm{L}$ of DADS for up to 24 hours, and finally stained with propidium iodide for cytofluorimetric analyses. The percentages of sub-G $\mathrm{G}_{1}$ (apoptotic) cells were calculated using WinMDI version 2.8 software. The cell cycle histograms reported are from one of five typical experiments done in triplicate that gave similar results.

of treatment with DADS followed by a recovery to control values at 24 hours. To confirm the involvement of ERK $1 / 2$ in the cell cycle arrest, we preincubated AGS cells with its specific inhibitor, U0126, for 1 hour. After 24 hours of treatment with DADS, cells were stained with propidium iodide for cytofluorimetric analyses. Figure $5 B$ shows that the inhibition of ERK $1 / 2$ induced an increase of the sub- $\mathrm{G}_{1}$ population, suggesting that the decision between proliferation and apoptosis is a process dependent, at least in part, on the reactivation of this member of the MAPK super family. Figure $5 B$ also reports the results obtained with SP600125, an inhibitor of JNK, as a control for specific involvement of ERK $1 / 2$ in the resistance to death. The selective inhibition of JNK induced per se a sustained and persistent arrest of cell cycle in $G_{2}-M$ phase, whereas in combination with DADS, it was able to decrease even the low amount of physiologic rate of apoptosis, confirming the key role of the constitutive activation of JNK in cell cycle re-entry.

Results thus far obtained show the involvement of both redox environment and ERK 1/2 modulation in the resistance and recovery from growth arrest upon DADS treatment. In order to identify a possible relationship between the two systems, we determined the trend of ERK $1 / 2$ phospho-activation in AGS cells previously depleted of GSH. Figure 6 shows that pretreatment for 12 hours with $1 \mathrm{mmol} / \mathrm{L}$ BSO inhibited the early decline of the phospho-active band of ERK $1 / 2$, allowing the commitment of cells to apoptosis. This phenomenon could also explain the decline of the phospho-active levels at 12 and 24 hours, suggesting a possible cross-talk between the intracellular redox state and ERK 1/2 modulation and, at the same time, it confirms the need for an early inactivation of this MAPK to produce cell cycle arrest and repair from DADS-mediated insult.

\section{Discussion}

Under physiologic conditions, the cell maintains a specific redox homeostasis that is mainly regulated by the GSSG/2GSH redox couple and to a lower extent by GS-R $(20,34)$. In this study, we have shown that AGS cells are resistant to DADS-mediated cytotoxic effects: the cells were only transiently arrested in $\mathrm{G}_{2}-\mathrm{M}$ phase as the result of induction of the p53/p21 system. We showed that AGS cells are characterized by a significantly higher content of GS-R with respect to the basal level, usually determined in tumor cell lines from different origins (data from our laboratory). Furthermore, these redox species seem to actively participate in the cell response against DADS-mediated cytotoxicity. In fact, GS-R levels increase concomitantly with cell growth inhibition and return to the basal content in concomitance with the cell cycle re-entry. Due to the transitory nature of the phenomenon observed, it might be suggested that GS-R formation represents an efficient buffering system among the early sensing cellular determinants of DADS effects. Additionally, the results indicate that cellular systems implicated in the reduction of GS-R (e.g., thioredoxin/thioredoxin reductase system) are operative and able to restore the basal conditions. Work is in progress in our laboratory in order to identify the mechanism(s) underlying the higher basal level of GS-R in AGS cells.

Under resting conditions, AGS cells show very high activity of the selenium-containing enzyme, GPx, which, in turn, is rapidly induced upon treatment with DADS. This response seems to

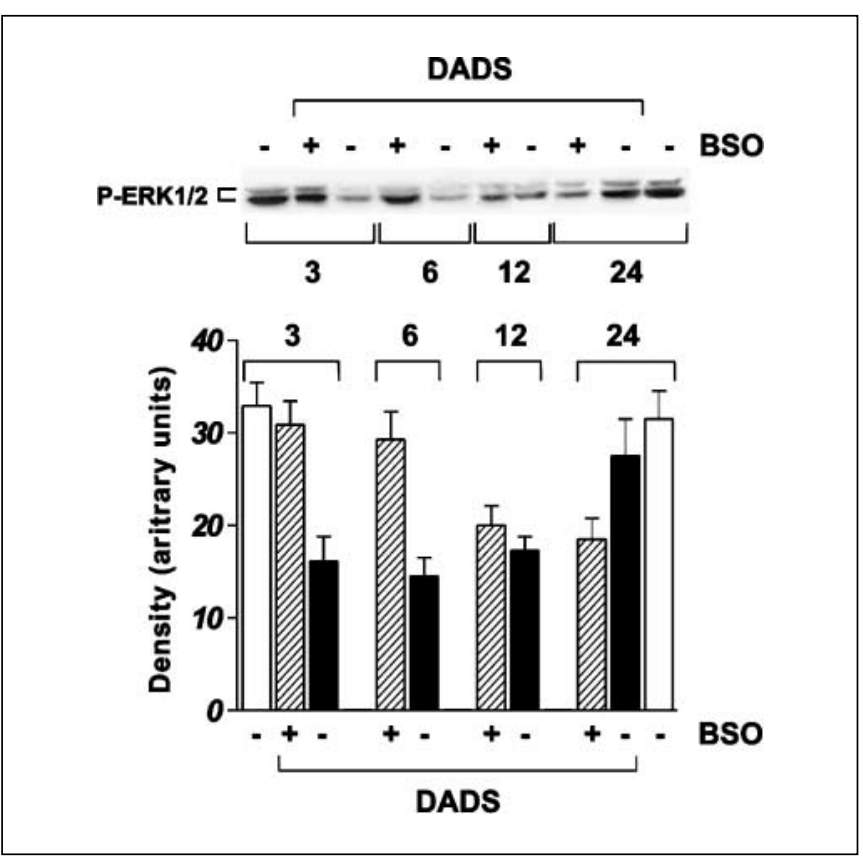

Figure 6. GSH depletion results in the maintenance of the phospho-active state of ERK 1/2. AGS cells were incubated with $1 \mathrm{mmol} / \mathrm{L}$ BSO for 12 hours and then treated with $50 \mu \mathrm{mol} / \mathrm{L}$ DADS up to 24 hours. Total cell extracts $(20 \mu \mathrm{g})$ were loaded for detection of the phospho-active isoform of ERK 1/2. The immunoblot is from one of five representative experiments that gave similar results.

Densitometric analyses of the immunoreactive bands were also done with FluorChem software (bottom). 
be highly efficient in counteracting the ROS-mediated insults because no detectable damages to lipids are envisaged under DADS challenge. These results are in line with the knowledge that gastric-derived cells are equipped with several GPx isoforms, among which, the gastrointestinal one, GI-GPx, has been shown to be up-regulated in colorectal adenomas and during neoplastic transformation (35).

The crucial role exerted by GSH in resistance to DADS-mediated insults was also confirmed by the experiments carried out in the presence of BSO, in which we showed that AGS cells could not buffer DADS-induced oxidative injuries when they are deprived of GSH. These results strongly suggest that the intrinsic capability of AGS cells to preserve protein thiols from irreversible oxidation, together with the high level and inducibility of GPx, could represent a specific tool of defense against oxidative insults. It could be interesting to identify the proteins that are specifically $S$-glutathiolated during DADS treatment, and whether this modification is functional for the modulation of protein activity.

Induction of cell cycle arrest upon DADS treatment seems to be necessary for cell response to its cytotoxicity. This was shown by the experiments carried out in the presence of both GSH-ethyl ester and selenomethionine, in which by increasing the intracellular ROS-buffering systems, cells efficiently metabolize DADS without any appreciable growth arrest.

It has been recently shown that diallyl trisulfide, a garlic-derived compound very similar to DADS, is able to induce the phosphoactivation of the histone H2A.X at $\mathrm{Ser}^{139}$, which controls the recruitment of the DNA repair machinery in response to DNA strand break during replication (36). These results, together with the evidence that under our experimental conditions, AGS cells are arrested between 12 and 24 hours of treatment without any concomitant measurable damages to proteins and lipids, reasonably suggest that DADS could be able to induce damage to DNA. The lipophilic nature of DADS, which confers the capability to freely cross cellular membranes, as well as the early activation of the p53/p21 system, lead us to suppose a direct harmful action of DADS, or DADS-derived radicals, within the nuclear compartment.

We previously showed that DADS induces apoptosis in neuroblastoma cells via ROS production and JNK/c-Jun pathway activation (21). Among the MAPK members, only ERK $1 / 2$ was involved in the response to DADS treatment. In particular, we observed a transient dephosphorylation of ERK $1 / 2$ concomitant to growth arrest and a subsequent reactivation necessary for cell cycle recovery. Indeed, AGS cells show very high levels of the basal phosphorylated isoform of both JNK and ERK $1 / 2$, which could be, as reported in literature, a consequence of the altered physiology of neoplastic cells (37). However, only the specific inhibition of ERK $1 / 2$ phosphorylation, by MEK $1 / 2$ upstream kinases, induces a significant increase of apoptotic cells, confirming the key role of ERK $1 / 2$ in AGS cell proliferation.

The MAPK signaling pathways seem to be profoundly affected by the intracellular redox environment. This is well-established for JNK and $\mathrm{p} 38^{\mathrm{MAPK}}$, the inhibitory proteins of which (glutathione$S$-transferase and thioredoxin, respectively) are redox-modulated $(38-40)$. The results obtained in this study, by the experiments carried out in the presence of BSO, show that the decrease of GSH levels affects ERK $1 / 2$ dephosphorylation upon DADS treatment, allowing us to speculate a possible modulation of this MAPK by the intracellular redox environment. Furthermore, GSH depletion commits cells to apoptosis in the presence of phospho-active ERK $1 / 2$, confirming the requirement of its dephosphorylation in order to enter cell cycle arrest and repair DADS-induced damages.

Overall, the results obtained indicate that chemotherapy based on the induction of oxidative stress cannot be indiscriminately applied for inducing apoptosis in cancer cells, but the pattern of antioxidants and MAPKs have to be taken into account to design specific treatments. Moreover, the results of the ERK $1 / 2$ signal transduction pathway confirm its deregulation in colorectal and gastric cancer cells and, at the same time, validate the potential of acting on this kinase as an approach to cancer treatment.

\section{Acknowledgments}

Received 8/26/2005; revised 9/20/2005; accepted 9/30/2005

Grant support: FIRB, MIUR, Consiglio Nazionale delle Ricerche, and Ministero della Sanità "Progetto di Ricerca Finalizzata."

The costs of publication of this article were defrayed in part by the payment of page charges. This article must therefore be hereby marked advertisement in accordance with 18 U.S.C. Section 1734 solely to indicate this fact.

\section{References}

1. Cadenas E, Davies KJ. Mitochondrial free radical generation, oxidative stress, and aging. Free Radic Biol Med 2000;29:222-30.

2. Bokoch GM, Knaus UG. NADPH oxidases: not just for leukocytes anymore! Trends Biochem Sci 2003;28:502-8.

3. Gonzalez FJ. Role of cytochromes $\mathrm{P} 450$ in chemical toxicity and oxidative stress: studies with CYP2E1. Mutat Res 2005;569:101-10.

4. Filomeni G, Rotilio G, Ciriolo MR. Disulfide relays and phosphorylative cascades: partners in redox-mediated signalling pathways. Cell Death Differ. Sep. 9;doi:10.1038/ sj.cdd. 4401754 .

5. Filomeni G, Rotilio G, Ciriolo MR. Cell signalling and the glutathione redox system. Biochem Pharmacol 2002; 64:1057-64.

6. Schafer FQ, Buettner GR. Redox environment of the cell as viewed through the redox state of the glutathione disulfide/glutathione couple. Free Radic Biol Med 2001; 30:1191-212.

7. Davies KJ, Delsignore ME, Lin SW. Protein damage and degradation by oxygen radicals: II. Modification of amino acids. J Biol Chem 1987;262:9902-7.

8. Gutteridge JM. Lipid peroxidation and antioxidants as biomarkers of tissue damage. Clin Chem 1995;41:1819-28.
9. Halliwell B, Aruoma OI. DNA damage by oxygenderived species. Its mechanism and measurement in mammalian systems. FEBS Lett 1991;281:9-19.

10. Matsuzawa A, Ichjio H. Stress-responsive protein kinases in redox-regulated apoptosis signaling. Antioxid Redox Signal 2005;7:472-81.

11. Sies H, Stahl W. Nutritional protection against skin damage from sunlight. Annu Rev Nutr 2004;24:173-200. 12. Nair J, Ohshima H, Nair Uj, Bartsch H. Endogenous formation of nitrosamines and oxidative DNA-damaging agents in tobacco users. Crit Rev Toxicol 1996;26:149-61. 13. Borek C. Antioxidant health effects of aged garlic extract. J Nutr 2001;131:1010-5S.

14. Frei B, Higdon JV. Antioxidant activity of tea polyphenols in vivo: evidence from animal studies. J Nutr 2003;133:3275-84S.

15. Sun SY, Hail N, Lotan R. Apoptosis as a novel target for cancer chemoprevention. J Natl Cancer Inst 2004;96: $662-72$.

16. Hassan HT. Ajoene (natural garlic compound): a new anti-leukaemia agent for AML therapy. Leuk Res 2004; 28:667-71.

17. Rotilio G, Mavelli I, Rossi L, Ciriolo MR. Biochemical mechanism of oxidative damage by redox-cycling drugs. Environ Health Perspect 1985;64:259-64.

18. Matsuzawa A, Nishitoh $H$, Tobiume $K$, Takeda $K$,
Ichijo H. Physiological roles of ASK1-mediated signa transduction in oxidative stress- and endoplasmic reticulum stress-induced apoptosis: advanced findings from ASK1 knockout mice. Antioxid Redox Signal 2002;4:415-25. 9. Adler V, Yin Z, Tew KD, Ronai Z. Role of redox potential and reactive oxygen species in stress signaling. Oncogene 1999;18:6104-11.

0. Filomeni G, Rotilio G, Ciriolo MR. Glutathione disulfide induces apoptosis in U937 cells by a redox-mediated p38 MAP kinase pathway. FASEB J 2003;17:64-6.

21. Filomeni G, Aquilano $\mathrm{K}$, Rotilio G, Ciriolo MR Reactive oxygen species-dependent c-Jun NH2-terminal kinase/c-Jun signaling cascade mediates neuroblastoma cell death induced by diallyl disulfide. Cancer Res 2003 63:5940-9.

22. Gupta N, Porter TD. Garlic and garlic-derived compounds inhibit human squalene monooxygenase. J Nutr 2001;131:1662-7.

23. Omkumar RV, Kadam SM, Banerji A, Ramasarma T. On the involvement of intramolecular protein disulfide in the irreversible inactivation of 3-hydroxy-3-methylglutaryl-CoA reductase by diallyl disulfide. Biochim Biophys Acta 1993;1164:108-12.

24. Pelicano H, Carney D, Huang P. ROS stress in cancer cells and therapeutic implications. Drug Resist Updat 2004;7:97-110. 
25. Hileman EO, Liu J, Albitar M, Keating MJ, Huang P. Intrinsic oxidative stress in cancer cells: a biochemical basis for therapeutic selectivity. Cancer Chemother Pharmacol 2003;53:209-19.

26. Kong Q, Beel JA, Lillehei KO. A threshold concept for cancer therapy. Med Hypotheses 2000;55:29-35.

27. Lewin MH, Arthur JR, Riemersma RA, et al. Selenium supplementation acting through the induction of thioredoxin reductase and glutathione peroxidase protects the human endothelial cell line EAhy926 from damage by lipid hydroperoxides. Biochim Biophys Acta 2000;1593:85-92.

28. Nicoletti I, Migliorati G, Pagliacci MC, Grignani F, Riccardi C. A rapid and simple method for measuring thymocyte apoptosis by propidium iodide staining and flow cytometry. J Immunol Methods 1991;139:271-9.

29. Brigelius R, Muckel C, Akerboom TP, Sies H Identification and quantitation of glutathione in hepatic protein mixed disulfides and its relationship to glutathione disulfide. Biochem Pharmacol 1983;32: 2529-34.

30. Rossi L, Ciriolo MR, Marchese E, De Martino A Giorgi M, Rotilio G. Differential decrease of copper content and of copper binding to superoxide dismutase in liver, heart and brain of copper-deficient rats. Biochem Biophys Res Commun 1994;203:1028-34.

31. Lowry OH, Rosebrough NJ, Farr AL, Randall RJ. Protein measurement with the Folin-phenol reagent. J Biol Chem 1951;193:265-75.

32. Chu FF, Esworthy RS, Doroshow JH. Role of Sedependent glutathione peroxidases in gastrointestina inflammation and cancer. Free Radic Biol Med 2004;36 1481-95.

33. Demeule M, Brossard M, Turcotte S, Regina A, Jodoin J, Beliveau R. Diallyl disulfide, a chemopreventive agent in garlic, induces multidrug resistance-associated protein 2 expression. Biochem Biophys Res Commun 2004; 324:937-45.
34. Finkel T. Reactive oxygen species and signal transduction. IUBMB Life 2001;52:3-6.

35. Banning A, Deubel S, Kluth D, Zhou Z, BrigeliusFlohe R. The GI-GPx gene is a target for Nrf2. Mol Cell Biol 2005;25:4914-23.

36. Herman-Antosiewicz A, Singh SV. Checkpoint kinase 1 regulates diallyl trisulfide-induced mitotic arrest in human prostate cancer cells. J Biol Chem 2005;280:28519-28. 37. Fang JY, Richardson BC. The MAPK signaling pathways and colorectal cancer. Lancet Oncol 2005;6:322-7. 38. Saitoh M, Nishitoh H, Fujii M, et al. Mammalian thioredoxin is a direct inhibitor of apoptosis signalregulating kinase (ASK) 1. EMBO J 1998;17:2596-606. 39. Adler V, Yin Z, Fuchs SY, et al. Regulation of JNK signaling by GSTp. EMBO J 1999;18:1321-34.

40. Filomeni G, Aquilano K, Rotilio G, Ciriolo MR Antiapoptotic response to GSH depletion: involvement of heat shock proteins and NF- $\kappa \mathrm{B}$ activation. Antioxid Redox Signal 2005;7:446-55. 